\title{
AN ARZELA-ASCOLI TYPE THEOREM FOR RANDOM FUNCTIONS
}

\author{
JOSEPH P. NOONAN and HENRY M. POLCHLOPEK \\ Department of Electrical Engineering \\ Tufts University \\ Medford, Massachusetts 02155 U.S.A. \\ (Received June 26, 1990 and in revised form March 6, 1991)
}

\begin{abstract}
A Theorem involving the convergence of random functions is presented. The Theorem is based upon the Arzela-Ascoli Theorem of Real Analysis. The development here may be useful in transient or asymptotic analysis of random functions and linear systems theory.
\end{abstract}

KEY WORDS AND PHRASES. Stochastic Processes, Arzela-Ascoli Theorem, Mean Square Convergence.

1980 Mathematics Subject Classification Codes: 60G07, 60G12

\section{INTRODUCTION.}

In this paper, we present some Theorems involving the mean squared convergence of sequences. They are based upon the Arzela-Ascoli Theorem of real analysis and are introduced in this mathematical format. Following this, we present two examples comparing our Theorem and Asymptotically WideSense Stationary (AWSS) sequences. The literature does not appear to have Theorems of this type, although considerable work has been done to collect theoretical results: Jacod and Shiryaev [1], Doob [2], Cramer and Leadbetter [3]. We feel these results will be useful in transient analysis applications and linear systems theory.

\section{THEORETICAL DEVELOPMENT.}

This is a generalization of the Arzela-Ascoli Theorem of real analysis to a certain class of random functions. We begin by stating the Arzela-Ascoli Theorem. Following which we consider some definitions and assumptions which will be needed in the statement and proof of the generalized theorem.

THEOREM 1 (Arzela Theorem). Every uniformly bounded equicontinuous family (sequence) of functions $\left(f_{1}(x), f_{2}(x), \ldots, f_{k}(x)\right)$ has at least one subsequence which converges uniformly on the $I[a, b]$.

We now consider some necessary concepts about random functions.

A random function is defined as a family $X(t)=(X(t), t \in T)$ of random variables on some probability space $(r, \lambda, \rho)$ where $r$ is a given space, $\lambda$ is the minimal completely additive class of sets defined over $r$, and $\rho$ is a probability measure function defined on the sets of $\lambda$.

We shall denote a family(sequence) of random functions $\left(X_{1}(t), X_{2}(t), \ldots, X_{k}(t)\right)$.

DEFINITION 1. A family of real random functions $\left(X_{1}(t), X_{2}(t), \ldots, X_{k}(t)\right)$ is uniformly bounded in mean square if there exists a $\beta \in R$ ( $\beta$ is finite) such that

$$
E\left\{\left|X_{n}(t)\right|^{2}\right\}<\beta
$$

for all $\mathrm{n} \geq 1$ and all $t \in T$.

Here $E$ is the Lebesgue Integral over the probability space. All Lebesgue Integrals are postulated to exist.

LEMMA 1. The autocorrelations $R_{n}(t, s)$ of a family of uniformly bounded in mean square random functions are a family of uniformly bounded functions on TxT.

PROOF. We have that

$$
E\left\{\left|X_{n}(t)\right|^{2}\right\}<\beta
$$


for all $n \geq 1$ and all $t \in T$. Therefore,

$$
R_{n}(t, s) \Delta E\left\{X_{n}(t) X_{n}(s)\right\}
$$

now we may use the Schwarz Inequality [4],

$$
\begin{gathered}
\leq\left[E\left\{X_{n}^{2}(t)\right\} E\left\{X_{n}^{2}(s)\right\}\right]^{1 / 2} \\
<[\beta * \beta]^{1 / 2}=\beta
\end{gathered}
$$

for all $n \geq 1$ and all $s, t \in T$. Therefore, $\left(R_{1}(t, s), R_{2}(t, s), \ldots, R_{n}(t, s)\right)$ is a uniformly bounded family of functions on TxT.

DEFINITION 2. A family of random functions is said to be equicontinuous in mean square (m.s.) sense, if for each $t \in T$ and $\varepsilon>0$, there exists a $\delta>0$ such that

$$
E\left\{\left|X_{n}(t)-X_{n}\left(t^{\prime}\right)\right|^{2}\right\}<e
$$

for all $n \geq 1$, whenever $\left|t-t^{\prime}\right|<\delta$.

DEFINITION 3. A family of functions is said to be equicontinuous if for each $t \in T$ and $\varepsilon>0$, there exists a $\delta>0$ such that

$$
\left|R_{n}(t, t)-R_{n}\left(t^{\prime}, t^{\prime}\right)\right|<e
$$

for all $n \geq 1$ whenever $\left|t-t^{\prime}\right|<\delta$.

LEMMA 2. The autocorrelations $R_{\mathrm{a}}(t, s)$ of a family of uniformly bounded in mean square equicontinuous (in m.s. sense) random functions is a family of equicontinuous functions on the line $t=t$ of TxT.

PROOF. Assume that the family of random functions $\left\{X_{q}(t)\right\}$ is equicontinuous in mean square sense. Consider $\left|R_{\mathrm{a}}(\mathrm{t}, \mathrm{t})-\mathrm{R}_{\mathrm{a}}\left(\mathrm{t}^{\prime}, \mathrm{t}^{\prime}\right)\right|$. Rearranging terms we get

$$
\begin{array}{r}
\left|R_{n}(t, t)-2 R_{n}\left(t, t^{\prime}\right)+R_{n}\left(t^{\prime}, t^{\prime}\right)+2 R_{n}\left(t, t^{\prime}\right)-2 R_{n}\left(t^{\prime}, t^{\prime}\right)\right| \\
\leq\left|R_{n}(t, t)-2 R_{n}\left(t, t^{\prime}\right)+R_{n}\left(t^{\prime}, t^{\prime}\right)\right|+2\left|R_{n}\left(t, t^{\prime}\right)-R_{n}\left(t^{\prime}, t^{\prime}\right)\right|
\end{array}
$$

We have that,

$$
E\left\{\left|X_{n}(t)-X_{n}\left(t^{\prime}\right)\right|^{2}\right\}<e
$$

for all $n \geq 1$ whenever $\left|t-t^{\prime}\right|<\delta$. Therefore,

$$
\left|R_{n}(t, t)-2 R_{n}\left(t, t^{\prime}\right)+R_{n}\left(t^{\prime}, t^{\prime}\right)\right|<e
$$

Now, $\left|R_{n}\left(t, t^{\prime}\right)-R_{n}\left(t^{\prime}, t^{\prime}\right)\right|$

$$
\begin{gathered}
=\left|E\left\{X_{n}(t) X_{n}\left(t^{\prime}\right)\right\}-E\left\{X_{n}\left(t^{\prime}\right) X_{n}\left(t^{\prime}\right)\right\}\right| \\
=\left|E\left\{X_{n}\left(t^{\prime}\right)\left(X_{n}(t)-X_{n}\left(t^{\prime}\right)\right)\right\}\right|
\end{gathered}
$$

and, again by the Schwarz Inequality,

$$
\leq\left[E\left\{X_{n}^{2}\left(t^{\prime}\right)\right\} E\left\{\left(X_{n}(t)-X_{n}\left(t^{\prime}\right)\right)^{2}\right\}\right]^{1 / 2}
$$

the first expectation is bounded by $\beta$. For the second expectation, we again apply our assumption, giving us that the above equation is

$$
\leq[\beta \cdot \epsilon]^{1 / 2}
$$

substituting these results back into Equation (2.1) we get 


$$
\left|R_{n}(t, t)-R_{n}\left(t^{\prime}, t^{\prime}\right)\right|<e+2 \beta^{1 / 2} e^{1 / 2} \Delta \epsilon_{0}
$$

where we have defined a new term $\varepsilon_{0}$. Note that,

$$
e=\left[\left(e_{0}+\beta\right)^{1 / 2}-\beta^{1 / 2}\right]^{2}
$$

Now, we have that for each $t \in T$ and $\varepsilon_{0}>0$, there exists a $\delta>0$ (Note: this is the same $\delta$ from the assumption) such that

$$
\left|R_{n}(t, t)-R_{n}\left(t^{\prime}, t^{\prime}\right)\right|<\mathbf{e}_{0}
$$

for all $n \geq 1$ whenever $\left|t-t^{\prime}\right|<\delta$. Therefore, the family of autocorrelations $\left\{R_{n}(t, t)\right\}$ is equicontinuous.

DEFINITION 4. A family (sequence) of random functions is said to converge in mean square (m.s.) sense to the random variable $X(t)$ if $E\left\{X_{n}^{2}(t)\right\}<\infty$ and $E\left\{X^{2}(t)\right\}<\infty$ and if

$$
\lim _{n \rightarrow \infty} E\left\{\left(X_{n}(t)-X(t)\right)^{2}\right\}=0
$$

With the above background at hand we now state and prove the main result of this paper.

THEOREM 2. Every uniformly bounded in mean square equicontinuous (in m.s. sense) family of random functions $\left(X_{1}(t), X_{2}(t), \ldots, X_{k}(t)\right)$ for $t \in T$ has at least one sub-sequence which converges in mean square sense on the $t \in T$.

PROOF. Given $\left(X_{1}(t), X_{2}(t), \ldots, X_{k}(t)\right)$ for $t \in T$ a family of uniformly bounded in mean square equicontinuous (in m.s. sense) random functions we have from Lemmas 1 \& 2 that:

The family $\left(R_{1}(t, s), R_{2}(t, s), \ldots, R_{t}(t, s)\right)$ on $T x T$ is a uniformly bounded equicontinuous family of functions along the line $t=t$ in TxT.

Thus, applying the Arzela-Ascoli Theorem to the family $\left(R_{1}(t, t), R_{2}(t, t), \ldots, R_{k}(t, t)\right)$ for $t \in T$, there exists a subsequence of $\left(R_{1}(t, t), R_{2}(t, t), \ldots, R_{k}(t, t)\right)$ which converges uniformly on $t=t$ in TxT. Let $\left(R_{m_{1}}(t, t), R_{m_{2}}(t, t), \ldots, R_{m_{k}}(t, t)\right)$ be this convergent subsequence. Let

$$
\lim _{i \rightarrow \infty} R_{m_{i}}(t, t)=R(t, t)
$$

That is, $R(t, t)$ is the limit of the subsequence.

Now, consider the subsequence of the family of random functions which corresponds to the above correlation functions. We denote this subsequence as

$$
\left(X_{m_{1}}(t), X_{m_{2}}(t), \ldots, X_{m_{k}}(t)\right)
$$

Note that this subsequence is also uniformly bounded in mean square and equicontinuous (in m.s. sense). Consider the expectation of any two terms of this subsequence. That is, $E\left\{X_{m_{i}}(t) X_{m_{j}}{ }_{j}(t)\right\}$ certainly,

$$
E\left\{X_{m_{1}}(t) X_{m_{j}}(t)\right\} \leq\left|E\left\{X_{m_{1}}(t) X_{m_{y}}(t)\right\}\right|
$$

And (yet again) by the Schwarz Inequality,

$$
\left|E\left\{X_{m_{1}}(t) X_{m_{j}}(t)\right\}\right| \leq\left[E\left\{X_{m_{1}}^{2}(t)\right\} E\left\{X_{m_{j}}^{2}(t)\right\}\right]^{1 / 2}
$$

And, because each subsequence is uniformly bounded in mean square the right hand side of the above equation is bounded by $[\beta \cdot \beta]^{1 / 2}=\beta$. Therefore,

$$
E\left\{X_{m_{1}}(t) X_{m_{j}}(t)\right\}<\beta
$$

Thus, the expectation is uniformly bounded.

Now consider,

$$
\left|R_{x_{m i}} x_{m, j}(t, t)-R_{x_{m y}} x_{m y}\left(t^{\prime}, t^{\prime}\right)\right|
$$

by again rearranging terms we get

$$
=\left|E\left\{X_{m_{1}}(t) X_{m_{j}}(t)\right\}-E\left\{X_{m_{1}}(t) X_{m_{j}}\left(t^{\prime}\right)\right\}+E\left\{X_{m_{1}}(t) X_{m_{j}}\left(t^{\prime}\right)\right\}-E\left\{X_{m_{1}}\left(t^{\prime}\right) X_{m_{j}}\left(t^{\prime}\right)\right\}\right|
$$




$$
\begin{aligned}
& =\left|E\left\{X_{m_{1}}(t)\left(X_{m_{j}}(t)-X_{m_{j}}\left(t^{\prime}\right)\right)\right\}+E\left\{\left(X_{m_{1}}(t)-X_{m_{1}}\left(t^{\prime}\right)\right) X_{m_{j}}\left(t^{\prime}\right)\right\}\right| \\
& \leq\left|E\left\{X_{m_{1}}(t)\left(X_{m_{j}}(t)-X_{m_{j}}\left(t^{\prime}\right)\right)\right\}\right|+\left|E\left\{\left(X_{m_{1}}(t)-X_{m_{1}}\left(t^{\prime}\right)\right) X_{m_{j}}\left(t^{\prime}\right)\right\}\right|
\end{aligned}
$$

Now, use the Schwarz Inequality one more time

$$
\begin{aligned}
\leq \quad & {\left[E\left\{X_{m_{1}}^{2}(t)\right\} E\left\{\left(X_{m_{j}}(t)-X_{m_{j}}\left(t^{\prime}\right)\right)^{2}\right\}\right]^{1 / 2} } \\
+ & {\left[E\left\{\left(X_{m_{1}}(t)-X_{m_{1}}\left(t^{\prime}\right)\right)^{2}\right\} E\left\{X_{m_{j}}^{2}\left(t^{\prime}\right)\right\}\right]^{1 / 2} }
\end{aligned}
$$

Next, use the equicontinuous (in m.s. sense) characteristics of these subsequences. That is, for each $t \in T$ and $\varepsilon>0$ there exists a $\delta>0$ such that the above equation

$$
\leq \beta^{1 / 2} e^{1 / 2}+e^{1 / 2} \beta^{1 / 2}=2 \beta^{1 / 2} e^{1 / 2} \Delta e_{1}
$$

Note: we have defined a new term $\varepsilon_{1}$ and $\varepsilon=\varepsilon_{1}{ }^{2} / 4 \beta$. Now, we have that for each $t \in T$ and $\varepsilon_{1}>0$ there exists a $\delta>0$ such that

$$
\left|R_{x_{m, j}} x_{m, j}(t, t)-R_{x_{m, 1}} x_{m y}\left(t^{\prime}, t^{\prime}\right)\right|<e_{1}
$$

for all $m_{i}, m_{\}} \geq 1$ whenever $\left|t-t^{\prime}\right|<\delta$. Therefore, the family of autocorrelations $\left\{R_{X_{m_{i}}} X_{m_{j}}(t, t)\right\}$ is equicontinuous. Therefore, $E\left\{X_{m_{i}}(t) X_{m_{j}}(t)\right\}$ is a uniformly bounded equicontinuous family of functions. Thus, we may apply the Arzela-Ascoli Theorem: the family $E\left(X_{m_{i}}(t) X_{m_{j}}(t)\right\}$ has at least one subsequence which converges uniformly. Call this subsequence $E\left\{X S_{a}(t) X S_{b}(t)\right\}$, where $a, b \in \operatorname{II}(\operatorname{Integers})$ and say it converges ot $R_{s s}(t, t)$. That is,

$$
\lim _{a, b \rightarrow \infty} E\left\{X S_{a}(t) X S_{b}(t)\right\}=R_{s s}(t, t)
$$

(Note: $R_{e s}(t, t)$ is certainly bounded by $R(t, t)$ and may or may not be the same function). In other words, the functions $E\left\{X S_{a}(t) X S_{b}(t)\right\}$ converge to a finite function on $T$ as $a, b \rightarrow \infty$.

We may now apply a theorem due to Loeve which proves our theorem. The theorem due to Loeve [5] can be stated as follows.

THEOREM 3 (Loeve). A uniformly bounded family of random functions converge in $T$ in mean square as $a \rightarrow \infty$ to some random function XS(t) on T (necessarily uniformly bounded) if and only if the functions $E\left(X S_{a}(t) X S_{b}(t)\right\}$ converge to a finite function on $T$ as $a, b \rightarrow \infty$.

This result finishes the proof of our Theorem 2 . We have already shown that $E\left\{X S_{a}(t) X S_{b}(t)\right\}$ converge to a finite function on $T$ as $a, b \rightarrow \infty$. Therefore, $X S_{2}(t)$ on $T$ converges in mean square as $a \rightarrow \infty$ to some random function XS( $(t)$. Therefore, there exists at least one subsequence which converges in mean square sense on the $t \in T$.

We may introduce a kind of "linearity" to this topic, in the sense that we consider a linear combination of our random variables and extend the above result. Formally, we write this as follows:

LEMMA 3. A linear combination of two uncorrelated families of random functions $\left(X_{1}(t), X_{2}(t), \ldots, X_{k}(t)\right)$ and $\left(Y_{1}(t), Y_{2}(t), \ldots, Y_{k}(t)\right)$, each of which is uniformly bounded in mean square and equicontinuous in mean square sense has at least one subsequence which converges in mean square sense on $t \in T$.

PROOF.

NOTE. In this proof and the next, the "(t)" is left out of the random variables in the expected value equations for the sake of brevity.

Again, by the Arzela-Ascoli Theorem, the family

$$
\left(R_{X_{1}}(t, t), R_{X_{2}}(t, t), \cdots, R_{X_{k}}(t, t)\right)
$$

has a uniformly convergent subsequence on $t=t$ in TxT. Call this convergent subsequence

$$
\left(R_{20 O_{1}}(t, t), R_{X x_{2}}(t, t), \cdots, R_{20 O_{k}}(t, t)\right)
$$


And

$$
\lim _{X M_{1} \rightarrow \infty} R_{x M_{i}}(t, t)=R_{X}(t, t)
$$

The subsequence of the family $\{X(t)\}$ of random functions corresponding to the above autocorrelations will be denoted

$$
\left(X_{n_{1}}(t), X_{n_{2}}(t), \cdots, X_{n_{k}}(t)\right)
$$

From the previous proofs (see Theorem 2) we know that the family $E\left(X_{n_{i}} X_{n_{j}}\right\}$ has at least one subsequence which converges uniformly, call this subsequence $\mathrm{E}\left\{\mathrm{XS}_{\mathrm{i}} \mathrm{XS} \mathrm{S}_{\mathrm{j}}\right\}$ where $\mathrm{i}, \mathrm{j} \in \mathrm{II}$ (Integers) and say it converges to $R_{x s}(t, t)$. That is,

$$
\lim _{i, j \rightarrow \infty} E\left\{X S_{1} X S_{j}\right\} \rightarrow R_{x S}(t, t)
$$

Likewise for the family $E\left\{Y_{m_{i}} Y_{m_{j}}\right\}$. That is,

$$
\lim _{i, j \rightarrow \infty} E\left\{Y S_{1} Y S_{j}\right\} \rightarrow R_{Y S}(t, t)
$$

Also, assume that the subsequences $\left\{X S_{1}(t)\right\}$ and $\left\{Y S_{1}(t)\right\}$ have means $u_{x}(t)$ and $u_{y}(t)$ respectively.

ASIDE: In actuality, to prove the Lemma, we need only that the means converge pointwise. However, often in stochastic processes applications a family of random functions will all have the same mean. So, keep in mind, the above assumption is sufficient, but not necessary. The Lemma statement has been left purposefully vague in this respect.

Now we may consider a linear combination. That is, for any constants a,b,c,de $\mathbf{R}$ and any integers $\mathrm{i}, \mathrm{j}, \mathrm{m}, \mathrm{n}$ we have that

$$
\begin{gathered}
E\left\{\left(a X S_{1}+b Y S_{m}\right)\left(c X S_{y}+d Y S_{n}\right)\right\} \\
=a c E\left\{X S_{1} X S_{y}\right\}+b d E\left\{Y S_{m} Y S_{n}\right\}+a d E\left\{X S_{1} Y S_{n}\right\}+b c E\left\{X S_{y} Y S_{m}\right\} \\
=a c E\left\{X S_{1} X S_{y}\right\}+b d E\left\{Y S_{m} Y S_{n}\right\}+a d \mu_{x}(t) \mu_{y}(t)+b C \mu_{x}(t) \mu_{y}(t)
\end{gathered}
$$

Taking limits as $\mathrm{i}, \mathrm{j}, \mathrm{m}, \mathrm{n} \rightarrow \infty$ the above equation converges to

$$
a c R_{x S}(t, t)+b d R_{Y S}(t, t)+(a d+b c) \mu_{x}(t) \mu_{y}(t)
$$

which is certainly a finite function. If we choose constants $a=c$ and $b=d$ then the above equation becomes

$$
a^{2} R_{X S}(t, t)+b^{2} R_{Y S}(t, t)+2 a b \mu_{x}(t) \mu_{y}(t)
$$

which is also a finite function. Therefore, from Loeve's Theorem, aXS $(t)+b Y S_{m}(t)$ on $T$ converges in mean square as $i, m \rightarrow \infty$ to some random function $Z(t)$. Therefore, there exists at least one subsequence which converges in mean square sense on the $t \in T$.

THEOREM 4 (Extension to $\mathrm{N}$ ). A linear combination of $\mathrm{N}$ uncorrelated families of random functions $\left(X 1_{1}(t), X 1_{2}(t), \ldots, X 1_{k}(t)\right),\left(X 2_{1}(t), X 2_{2}(t), \ldots, X 2_{k}(t)\right), \ldots,\left(X N_{1}(t), X N_{2}(t), \ldots, X N_{k}(t)\right)$ each of which is uniformly bounded in mean square and equicontinuous in mean square sense, has at least one subsequence which converges in mean square sense on $t \in T$.

PROOF. We will use proof by induction. Assume this holds for $n$. That is, assume that a linear combination of $n$ uncorrelated families of random functions each of which is uniformly bounded in mean square and equicontinuous in mean square sense has at least one subsequence which converges in mean square sense on $t \in T$. Let us call this convergent subsequence

$$
\left(X_{m_{1}}(t), X_{m_{2}}(t), \ldots, X_{m_{k}}(t)\right)
$$


From the previous proofs we know that the family $\mathrm{E}\left(\mathrm{X}_{\mathrm{m}_{\mathrm{i}}} \mathrm{X}_{\mathrm{m}_{\mathrm{j}}}\right\}$ has at least one subsequence which converges uniformly, call this subsequence $E\left(X S_{1} X S_{j}\right)$ where $i, j$ are integers and say it converges to $\mathbf{R}_{\mathbf{x s}}(\mathrm{t}, \mathrm{t})$. That is,

$$
\lim _{i, j \rightarrow \infty} E\left\{x S_{1} X S_{j}\right\} \rightarrow R_{x s}(t, t)
$$

Next consider the $n+l$ case. That is, consider another family $\left(Z_{1}(t), Z_{2}(t), \ldots, Z_{k}(t)\right)$ that meets the same criterion as above. Likewise for the family $E\left\{Z_{p_{i}} Z_{p_{j}}\right\}$. That is,

$$
\lim _{i, j \rightarrow \infty} E\left\{z S_{1} z S_{j}\right\} \rightarrow R_{z S}(t, t)
$$

Also, assume that the subsequences $\left\{X S_{1}(t)\right\}$ and $\left\{Z S_{1}(t)\right\}$ have means $u_{x}(t)$ and $u_{z}(t)$ respectively.

NOTE. The aside from Lemma 3 also applies here for the proof of this theorem.

Consider a linear combination of these subsequences. That is, for $a, b, c, d \in \mathbf{R}$ and integers $i, j, m, n$ we have

$$
\begin{gathered}
E\left\{\left(a X S_{1}+b Z S_{m}\right)\left(c X S_{y}+d Z S_{n}\right)\right\} \\
=a c E\left\{X S_{1} X S_{y}\right\}+b d E\left\{Z S_{m} Z S_{n}\right\}+a d E\left\{X S_{1} Z S_{n}\right\}+b c E\left\{X S_{y} Z S_{m}\right\}
\end{gathered}
$$

Now, by assumption, the new family $\left\{Z_{i}(t)\right\}$ is uncorrelated to each and every one of the $n$ families that make up the convergent subsequence $\left\{X S_{1}(t)\right\}$. Thus, I claim that the family $\left\{Z_{i}(t)\right\}$ and the family $\left\{X S_{i}(t)\right\}$ are uncorrelated. Therefore, we have

$$
E\left\{X S_{1} Z S_{n}\right\}=E\left\{X S_{y} Z S_{m}\right\}=\mu_{x}(t) \mu_{z}(t)
$$

and equation (2.2) becomes

$$
a c E\left\{X S_{1} X S_{y}\right\}+b d E\left\{Z S_{m} Z S_{n}\right\}+a d \mu_{x}(t) \mu_{z}(t)+b C \mu_{x}(t) \mu_{z}(t)
$$

Taking limits as $\mathrm{i}, \mathrm{j}, \mathrm{m}, \mathrm{n} \rightarrow \infty$ the above equation converges to

$$
a c R_{x S}(t, t)+b d R_{z S}(t, t)+(a d+b c) \mu_{x}(t) \mu_{z}(t)
$$

which is certainly a finite function. If we choose constants $a=c$ and $b=d$ then the above equation becomes

$$
a^{2} R_{x S}(t, t)+b^{2} R_{z S}(t, t)+2 a b \mu_{x}(t) \mu_{z}(t)
$$

which is also a finite function. Again, by Loeve's Theorem we know that $a X S_{i}(t)+b Z S_{m}(t)$ on $T$ converges in mean square as $i, m \rightarrow \infty$ to some random function $X_{0}(t)$. Therefore, there exists at least one subsequence which converges in mean square sense on the $t \in T$.

The proof of Theorem 4 is now complete. That is, we have shown it is true for $n=1$ (Theorem 2) and having assumed it true for $n$, shown it to be true for $n+1$ (by the above proof). QED

\section{APPLICATIONS AND EXAMPLES}

This research may be applied to linear systems theory. Let us consider a basic dynamical equations description

$$
y(t)_{n \times 1}=[A]_{m \times n} x(t)_{n \times 1}
$$

where, as indicated, $x(t)$ is an $n x 1$ vector(input), $y(t)$ is an $m x 1$ vector(output) and [A] is an mxn matrix with constant coefficients. Assume that each of the $n$ components (families) of the input vector $x(t)$ is uniformly bounded in mean square and equicontinuous in mean square sense, and the $n$ components are uncorrelated. Then, by Theorem 4, we know that each of the m components (families) of the output vector $y(t)$, has at least one subsequence which converges in mean square sense on $t \in T$. This may have applications concerning the uniform asymptotic stability of systems (Chen [6]).

Next, we look at two examples.

EXAMPLE 1 (Stark and Woods [7]). As an example of this Theorems' application, consider the mean square differential equation 


$$
\frac{d Y(t)}{d t}+\alpha Y(t)=X(t) \quad t \geq 0
$$

Assume $\alpha>0$. Let $X(t)$ be a stationary process with mean $u_{x}$ and convariance function $R_{x x}(\tau)=\sigma^{2} \delta(\tau)$. Let $Y(t)$ be the solution subject to the initial condition $Y(0)=0$, we obtain the covariance solution

$$
R_{Y Y}(t, s)=\frac{\sigma}{2 \alpha}(\exp (-\alpha s))(\exp (\alpha t)-\exp (-\alpha t)) \quad 0<t s s
$$

And,

$$
R_{Y Y}(t, s)=\frac{\sigma}{2 \alpha}(1-\exp (-2 \alpha s))(\exp (-\alpha(t-s)) \quad t \geq s
$$

This covariance function solution to the mean square differential equation also satisfies our Theorem 2 (That is, $\mathrm{Y}(\mathrm{t})$ is uniformly bounded in mean square and equicontinuous in the mean square sense).

Now, we provide a natural extension to a family of such functions.

Consider a family of random functions $\left(Y_{1}(t), Y_{2}(t), \ldots, Y_{k}(t)\right)$ where, for $1 \leq i \leq k, X_{i}(t)$ has mean $u_{x_{j}}$ and covariance function $R_{x_{i}} x_{i}(\tau)=\sigma_{i}^{2} \delta(\tau)$ and $Y_{i}(t)$ is the solution to the mean square differential
equation

$$
\frac{d Y_{1}(t)}{d t}+\alpha Y_{1}(t)=X_{1}(t) \quad t \geq 0
$$

Then from the above argument and Theorem 2, this family of random function solutions $\left(Y_{1}(t), Y_{2}(t), \ldots, Y_{k}(t)\right)$ has at least one subsequence which converges in mean square sense on the $t \in T$.

Also, we know that for any fixed $\tau$,

$$
\lim _{t \rightarrow \infty} R_{Y Y}(t+\tau, t)=\frac{\sigma^{2}}{2 \alpha} \exp (-\alpha|\tau|)
$$

This means that $Y(t)$ is asymptotically wide-sense stationary (AWSS).

EXAMPLE 2 (Parzen [8]). Consider the oscillator with fluctuating frequency:

$$
x(t)=\cos \left(\varpi_{0} t+\phi(t)\right) \quad t \geq 0
$$

where for $t \neq s$, we have covariance

$$
\begin{aligned}
R_{x x}(t, t)= & \frac{1}{2} \cos \left(\varpi_{0}(t-s)\right) \exp (-\alpha(t-s) / 2) \\
& +\frac{1}{2} \cos \left(\varpi_{0}(t+s)\right) \exp (-\alpha(t+s) / 2)
\end{aligned}
$$

and for $\mathrm{t}=\mathrm{s}$, we have

$$
R_{x x x}(t, t)=\frac{1}{2}+\frac{1}{2} \cos \left(2 \varpi_{0} t\right) \exp (-4 \alpha t / 2)
$$

This does not satisfy the criterion of our Theorem 2. Specifically $X(t)$ is not equicontinuous in mean square sense. However, this process $\mathrm{X}(\mathrm{t})$ is asymptotically wide sense stationary because,

$$
\lim _{t \rightarrow \infty} R_{x x}(t+\tau, t)=\frac{1}{2} \cos \left(\omega_{0} t\right) \exp (-\alpha \tau / 2)
$$

We have here two examples of AWSS processes, one can be applied to Theorem 2, the other cannot. More work needs to be done to discover the connection (if any) between this mean square convergent subsequence and AWSS processes. It is clear that the theorem provides a sufficient but not necessary condition for convergence. 


\section{References}

[1] Jean Jacod and Albert N. Shiryaev, Limit Theorems For Stochastic Processes, Springer-Verlag, Heidelberg, 1987.

[2] Joseph L. Doob, Stochastic Processes, Wiley, 1953.

[3] Harald Cramer and M.R. Leadbetter, Stationary and Related Stochastic Processes, Wiley, 1967, Ch.5.

[4] Robert M. Gray and Lee D. Davisson, Random Processes: A Mathematical Approach for Engineers, Prentice-Hall, 1986, pp154.

[5] Michel Loeve, Probability Theory, Van Nostrand, 1963, pp469.

[6] Chi-Tsong Chen, Linear System Theory and Design, Holt, Rinehart and Winston, 1984, pp403.

[7] Henry Stark and John W. Woods, Probability Random Processes, and Estimation Theory for Engineers, Prentice-Hall, 1986, pp.314-315.

[8] Emanuel Parzen, "Spectral Analysis of Asymptotically Stationary Time Series", Bull. Inst. Internal. Statist. 39, 1962, pp.87-103. 


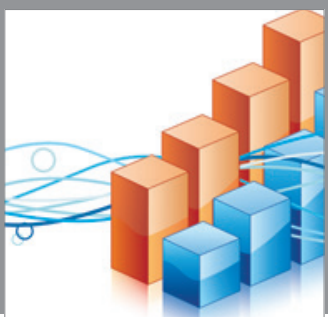

Advances in

Operations Research

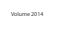

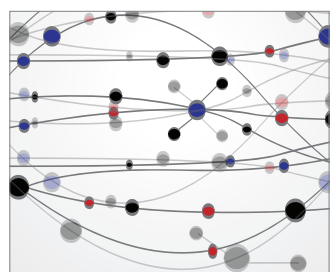

\section{The Scientific} World Journal
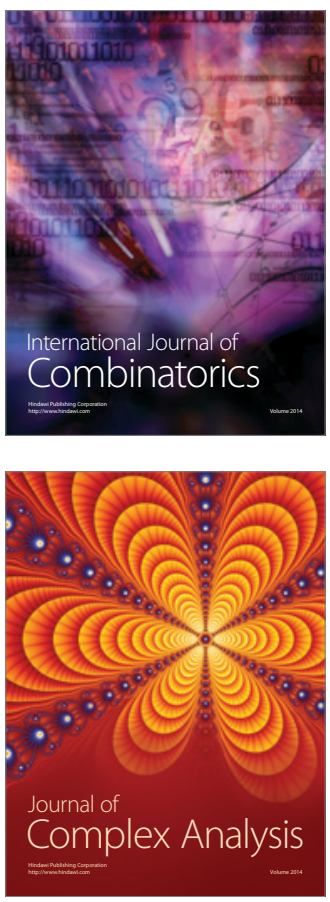

International Journal of

Mathematics and

Mathematical

Sciences
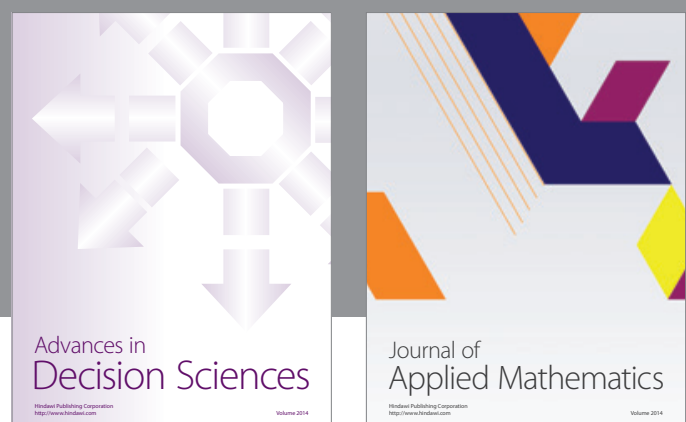

Journal of

Applied Mathematics
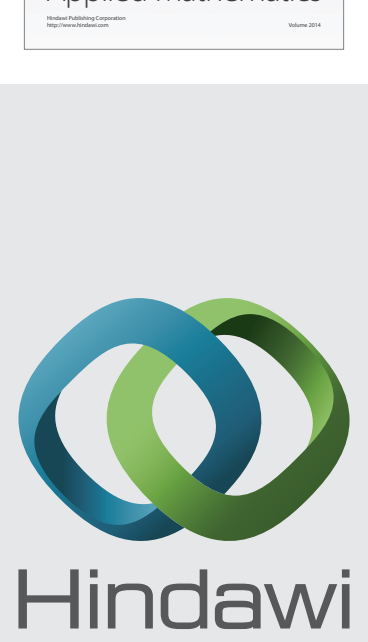

Submit your manuscripts at http://www.hindawi.com
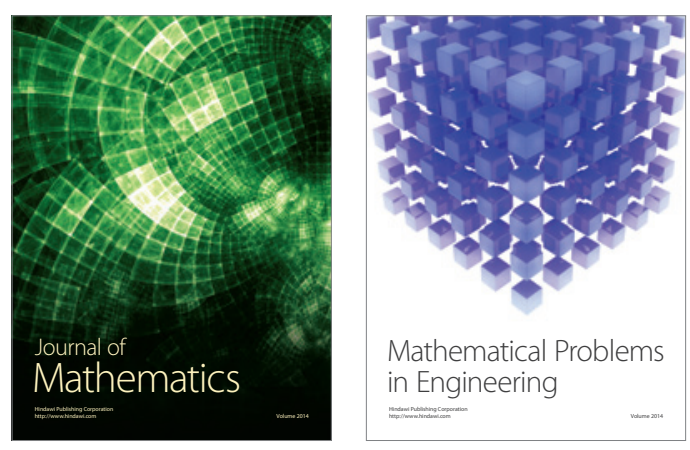

Mathematical Problems in Engineering
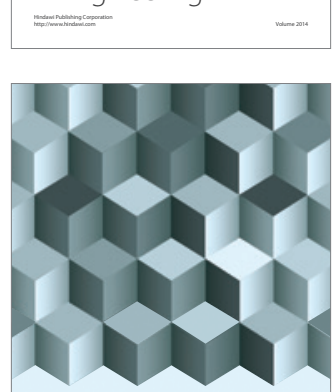

Journal of

Function Spaces
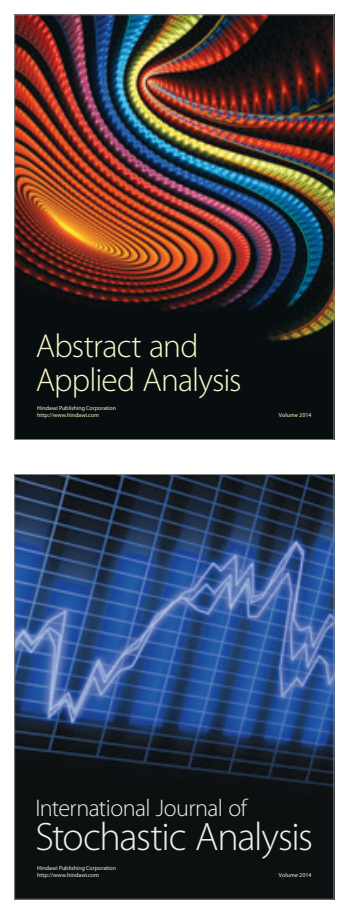

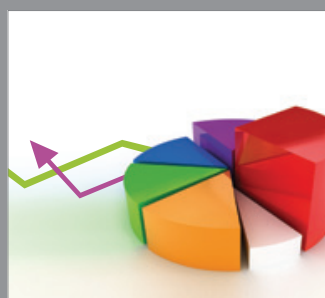

ournal of

Probability and Statistics

Promensencen
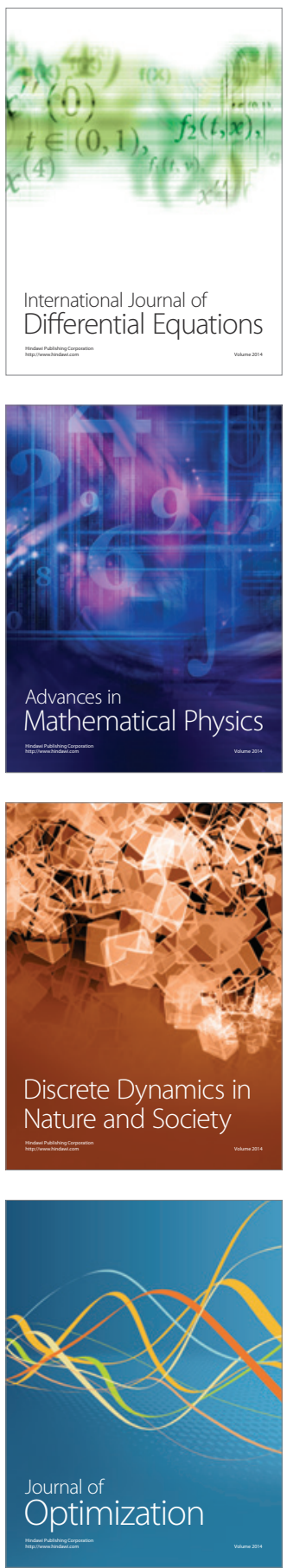